\title{
HYBRID ENERGY-AWARE SYNCHRONIZATION ALGORITHM IN WIRELESS SENSOR NETWORKS
}

\author{
Robert Akl \\ University of North Texas \\ Denton, Texas, US
}

\section{ABSTRACT}

We present a time synchronization scheme for wireless sensor networks that aims to conserve sensor battery power while maintaining network connectivity for as long as possible. The proposed method creates a hierarchical tree by flooding the sensor network from a designated source point. It then uses a hybrid algorithm derived from the Timing-sync Protocol for Sensor Networks (TSPN) and the Reference Broadcast Synchronization method (RBS) to periodically synchronize sensor clocks by minimizing the number of required transmissions. In multi-hop ad-hoc networks, a depleted sensor will drop information from all other sensors that route data through it, decreasing the physical area being monitored by the network. It is therefore imperative that time synchronization schemes are aware of the number of sensors being used at any given time. The proposed method uses several techniques and thresholds to maintain network connectivity. A new source point is chosen when the current one's battery power reaches a designated energy threshold. The network is also re-flooded whenever the number of used sensors drops below another threshold. We implement and show that our scheme can provide significant power savings over both TPSN and RBS; the power reduction is even more drastic in large multi-hop sensor networks. The method also improves upon these algorithms by maintaining a large area of coverage even when some sensors lose power.

\section{INTRODUCTION}

Over the past few years, applications have been developed to monitor environmental properties such as temperature and humidity; they can also be used to analyze motion of animals or vehicles. One of the most important requirements for these monitoring applications is unobtrusiveness; this creates a need for wireless ad-hoc networks using very small sensing nodes. These special networks are called "wireless sensor networks" or WSNs. These networks are built from many wireless sensors in a high-density configuration to provide redundancy and to monitor a large physical area.

Time synchronization is a crucial aspect of any networked system. The majority of research in this field has concentrated on traditional high-speed computer networks with few power restraints, leading to the Global Positioning System (GPS) [1] and the Network Time Protocol (NTP) [2]. These conventional networks are effective for communication of large amounts of data, typical of Internet and Local Area Networks (LANs).

With the emergence of WSNs, current LAN synchronization methods will not work efficiently. GPS provides good synchronization accuracy, but requires a very large amount of power from the sensors. In a power-

\author{
Yanos Saravanos \\ University of North Texas \\ Denton, Texas, US
}

constrained sensor, this synchronization is infeasible. NTP is also infeasible since it is designed for traditional computer networks and will not scale well for wireless sensor networks. Some new synchronization methods have been developed specifically for sensor networks, such as the Reference Broadcast Synchronization method (RBS) [3] and the Timing-sync Protocol for Sensor Networks (TPSN) [4], [5].

RBS and TPSN both achieve accurate clock synchronization within a few microseconds of uncertainty. However, they are both designed for networks with a small number of sensors and are not specifically geared towards energy conservation; although these algorithms will work for larger networks, their energy consumption becomes inefficient and network connectivity is not maintained once nodes begin losing power. Simulating each of these methods shows that synchronizing a large sensor network requires an unnecessarily large number of transmissions, which will quickly deplete sensors and reduce the network's coverage area.

This work concentrates on the following aspects of WSNs:

1. Design a hybrid method between RBS and TPSN to reduce the number of transmissions required to synchronize an entire network.

2. Extend single-hop synchronization methods to operate in large multi-hop networks.

3. Verify that the hybrid method operates as desired by simulating against RBS and TPSN.

4. Maintain network connectivity and coverage.

The rest of the paper is organized as follows. In section II, we present an overview of previous time synchronization algorithms including RBS and TPSN. In section III, we design our hybrid synchronization algorithm that is also energy-aware. Results are given in section IV. Finally, section $\mathrm{V}$ concludes the paper.

\section{WSN TIME SYNCHRONIZATION ALGORITHMS}

Although traditional synchronization methods are effective for computer networks, they are ineffective in sensor networks. New synchronization algorithms specifically designed for wireless sensor networks have been developed and can be used for several applications [6]. In [7], the authors present a probabilistic method for clock synchronization based on RBS. In [8], the authors present a level-based and a diffusion-based clock synchronization that is resilient to compromise of some source nodes. In [9], the authors describe four methods to achieve global synchronization: a node-based, a hierarchical cluster-based, a diffusion-based, and a fault-tolerant based approach. In [10], the authors propose creating spanning trees with multiple subtrees, in which two subtree synchronization algorithms can be performed. In [11], the authors propose E-RBS (Efficient 
RBS) to decrease the number of messages to be processed and save energy consumption within a given accuracy range.

\section{A. $R B S$}

One of the first major research attempts to create a time synchronization algorithm specifically tailored for sensor networks led to the development of Reference Broadcast Synchronization (RBS) in 2002 [3]. This algorithm defines the critical path, which is the portion of the network where a significant amount of clock uncertainty exists. A long critical path results in high uncertainty and low accuracy in the synchronization. There are four main sources of delays that must be accounted for to have accurate time synchronization:

- Send time: this is the time to create the message packet.

- Access time: this is a delay when the transmission medium is busy, forcing the message to wait.

- Propagation time: this is the delay required for the message to traverse the transmission medium from sender to receiver.

- Receive time: similar to the send time, this is the amount of time required for the message to be processed once it is received.

The RBS algorithm can be split into three major events:

1. Flooding: a transmitter broadcasts a synchronization request packet.

2. Recording: the receivers record their local clock time when they initially pick up the sync signal from the transmitter.

3. Exchange: the receivers exchange their observations with each other.

There are two unique implementations of RBS. The simplest method is designed for very high accuracy for sparse networks, where transmitters have at most two receivers. The transmitter can broadcast a synchronization request to the two receivers, which will record the times at which they receive the request, just as the algorithm describes. However, the receivers will exchange their observations with each other multiple times, using a linear regression to lower the clock offset.

The other version of the RBS algorithm involves the following steps: the transmitter sends a reference packet to two receivers; each receiver checks the time when it receives the reference packet; the receivers exchange their recorded times. The main problems with this scheme are the nondeterminism of the receiver, as well as clock skew. The receiver's non-determinism can be resolved by simply sending more reference packets. The clock skew is resolved by using the slope of a least-squares linear regression line to match the timing of the crystal oscillators.

RBS can be adapted to work in multi-hop environments as well. Assuming a network has grouped clusters with some overlapping receivers, linear regression can be used to synchronize between receivers that are not immediate neighbors. However, it is more complicated than the singlehop scenario since there will be timestamp conversions as the packet is relayed through nodes. This extra complication is manifested in larger synchronization errors.

B. TPSN

The Timing-Sync Protocol for Sensor Networks (TSPN) was developed in 2003 [4] in an attempt to further refine time synchronization beyond RBS's capabilities. TPSN uses the same sources of uncertainty as RBS does (send, access, propagation, and receive), with the addition of two more:

- Transmission time: the time for the packet to be processed and sent through the RF transceiver during transmission.

- Access time: the time for each bit to be processed from the RF transceiver during signal reception.

The TPSN works in two phases:

1. Level Discovery Phase: this is a very similar approach to the flooding phase in RBS, where a hierarchical tree is created beginning from a root node.

2. Synchronization Phase: in this phase, pair-wise synchronization is performed between each transmitter and receiver.

In the level discovery phase, each sensor node is assigned a level according to the hierarchical tree. A pre-determined root node is assigned as level 0 and broadcasts a level_discovery packet. Sensors that receive this packet are assigned as children to the transmitter and are set as level 1 (they will ignore subsequent level_discovery packets). Each of these nodes broadcasts a level_discovery packet, and the pattern continues with the level 2 nodes.

TPSN is a great improvement over RBS in terms of accuracy. Using a 2-way handshake reduces uncertainty in half since the average of the time differences is used.

The main disadvantage that TPSN faces is its energy consumption in sparse networks; a 2-way handshake requires each node to receive a packet and to send one in response. For a parent node $A$ with two children $B$ and $C$, node $A$ broadcasts the level_discovery packet, and then a synchronization_pulse packet. Nodes $B$ and $C$ receive both packets, and then transmit an ack packet back to node $A$. This example uses 4 transmissions and 4 receptions for TPSN (to do both level discovery and synchronization) ${ }^{1}$. In contrast, the same situation would only require 2 transmissions and 3 receptions when using RBS; node $A$ broadcasts a synchronization request packet with a timestamp, and then node $B$ sends a second transmission to node $C$ with its observation (node $C$ can also transmit to node $B$ with the same end result thus requiring 3 transmissions and 4 receptions).

In addition, TPSN has the same problem as RBS with respect to lost network coverage when nodes begin losing power. A dead transmitter node will drop all of its receivers from the network, lowering the WSN's coverage area. Network restructuring is not included in the TPSN algorithm.

\footnotetext{
${ }^{1}$ Note that in a static sensor network if the level discovery process is not launched, for one round of synchronization, it only requires 2 transmissions and 2 receptions.
} 
RBS and TPSN are some of the first efforts in creating synchronization algorithms tailored towards low-power sensor networks. They both have unique strengths when dealing with energy consumption. RBS is most effective in networks where transmitting sensors have few receivers, while TPSN excels when transmitters have many receivers

\section{ENERGY-AwARE TIME SYNCHRONIZATION}

In this section, we present our hybrid synchronization algorithm.

\section{A. Hybrid Flooding}

Before the sensors can be synchronized, a network topology must be created. Algorithm 1 is used by each sensor node to efficiently flood the network.

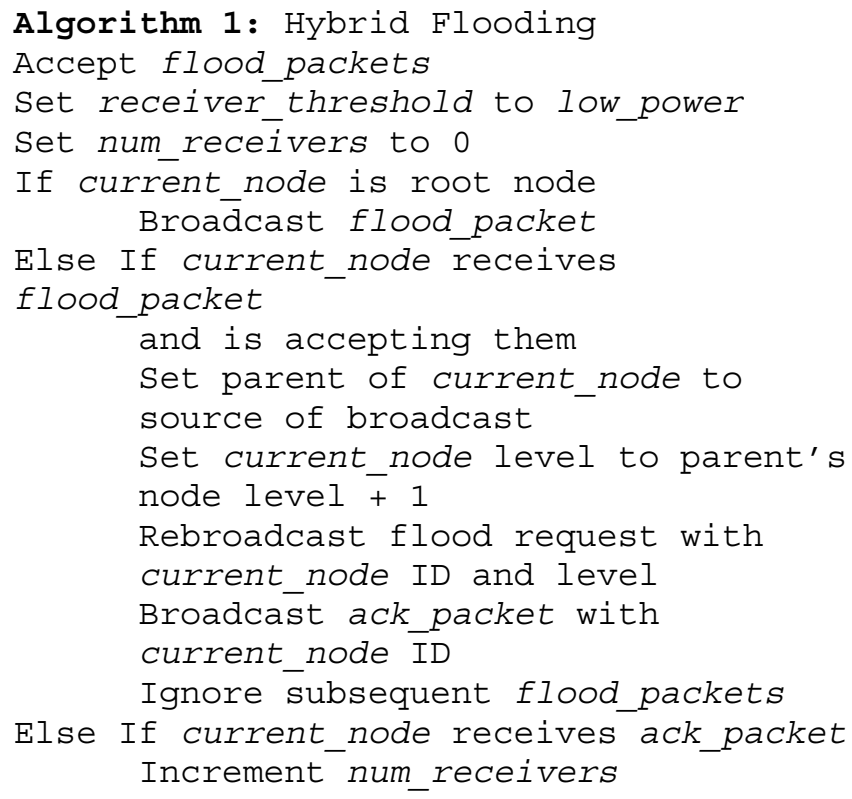

Each sensor is initially set to accept flood_packets, but will ignore subsequent ones in order not to be continuously reassigned as the flood broadcast propagates. The num receivers variable keeps track of the node's receivers and is used in the synchronization algorithm.

\section{B. Hybrid Synchronization}

Once the network flooding has been completed, the network can be synchronized using the determined hierarchy. In networks where the sensors are dispersed at random, there will be patches of high density node distribution interspersed with lower density regions. A transmitter in a high density area will usually have a large number of receivers, while another transmitter in a lower density section will usually have 1 or 2 receivers at most.

The hybrid algorithm minimizes power regardless of the network's topology by choosing the best synchronization technique depending on the number of children connected to the transmitter. Since the energy required for reception usually differs from that of a transmission, the ratio of the reception power to the transmission power is needed in order to find the optimal point at which to switch from receiverreceiver synchronization to transmitter-receiver synchronization

Algorithm 2 describes the algorithm used for the hybrid synchronization.

Algorithm 2: Hybrid Synchronization set receiver threshold to high power If num_receivers < receiver_threshold // Use $R B \bar{S}$ algorithm

Transmitter broadcasts sync_request For each receiver

Record local time of

reception for sync request Broadcast observation packet Receive observation_packet from other receivers

Else // Use TPSN algorithm

Transmitter broadcasts sync_request For each receiver

Record local time of reception for sync request Broadcast ack_packet to transmitter with local time

\section{Energy Depletion}

Another issue that the hybrid algorithm addresses when synchronizing a sensor network is the effect that a depleted sensor has on the topology. Once the battery is exhausted, the node will be dropped from the network, but so will all of the receivers depending on it. This loss of connectivity cascades through each receiver, so a drastic restructuring can occur when a high-level sensor is drained. The hybrid algorithm keeps track of the number of powered nodes. Once this number decreases below a user-defined threshold, the network is re-flooded using Algorithm 1. Should the source node lose power, a new source node is chosen from the original one's receivers. These receivers communicate their power levels with each other and the one with the most remaining energy is elected as the new root node, as show in Algorithm 3.

Algorithm 3: Root Node Election If cur_node_level $==1$ and cur_node_power allows 1 more $\mathrm{TX}$

Broadcast elect_packet with cur node ID

If cur note level $==2$

Broadcast elect_packet with cur_node_ID, cur_node_power If cur_node receìives elect_packet and elect_packet_power >= cur_node_power set elect_packet_ID to root node

In addition, receivers will only analyze the sync_request packets from their respective transmitters when using the 
TPSN-style synchronization. This saves additional battery power since the receivers do not have to analyze packets they overhear from other broadcasting transmitters.

Lastly, the dropped packets are monitored. This is a useful statistic since it keeps track of algorithm efficiency and wasted energy. Dropped packets also allow us to compare various network topologies and determine which ones allow for the most energy conservation.

Our hybrid algorithm has the same order of implementation complexity and fairness as RBS and TPSN.

\section{RESUlts AND ANALYSIS}

\section{A. Hybrid Algorithm Validation}

Several simulations were run (in MATLAB) to compare our hybrid algorithm with RBS and TPSN. A transmitting sensor can dynamically switch between RBS and TPSN by simply comparing the number of connected receivers to the reception/transmission power ratio. Twenty simulations are run over a $1000 \mathrm{~m} \times 1000 \mathrm{~m}$ area which is randomly populated with 500 sensors, and the path loss coefficient is set to 3.5 . In each simulation, the receiver_threshold value is changed from 1 to the largest number of receivers connected to a sensor. The hybrid synchronization algorithm is executed for each of these receiver_threshold values and the energy consumption is stored and compared to the consumption of TPSN, RBS, and the hybrid synchronization algorithm. Each of the data points is plotted, along with a line representing the average from all of the simulations. For the MICA2Dot platform [12], a reception uses approximately $24 \mathrm{~mW}$ of power, while a transmission requires $75 \mathrm{~mW}$ at $-5 \mathrm{dBm}$.

The hybrid algorithm uses the least amount of energy when the receiver threshold is set to 4.42. This means that transmitters with 4 or fewer sensors will use RBS for synchronization while those with 5 or more receivers will use TPSN. Fig. 1 illustrates how changes in the receiver_threshold value affect the hybrid algorithm. The energy consumption from the hybrid algorithm when using the optimal receiver threshold value is lower than both TPSN and RBS. The minimum value is found between values of 4 and 5. Lastly, the spread amongst data points increases dramatically as the receiver threshold increases beyond 13 .

More importantly, setting the receiver threshold value to 1 will force a transmitter to use TPSN. The hybrid algorithm in this case will have the same energy consumption as TPSN. On the other hand, a receiver_threshold set to the largest number of receivers connected to a transmitter will force a transmitter to use RBS.

The hybrid synchronization algorithm is very dynamic and will adapt itself to multiple equipment specifications. The power requirements for the MicaZ [13] sensor platform are different from the Mica2DOT platform; MicaZ uses $59.1 \mathrm{~mW}$ for a reception, but only uses $42 \mathrm{~mW}$ for each transmission at $-5 \mathrm{dBm}$. When using MicaZ, the optimal receiver threshold value is 3.42 as shown in Fig. 2, where the local minimum has shifted further to the left when compared to the Mica2DOT platform. Even though the value of the receiver threshold should be calculated each time the network platform changes, both of the above examples yield somewhat similar values for the optimal receiver threshold.

\section{B. Power Consumption}

The next set of simulations demonstrates the algorithm's reduction in power consumption for several network sizes. The number of sensors is changed from 250 up to 1500 , in increments of 250. The Mica2DOT platform is used.

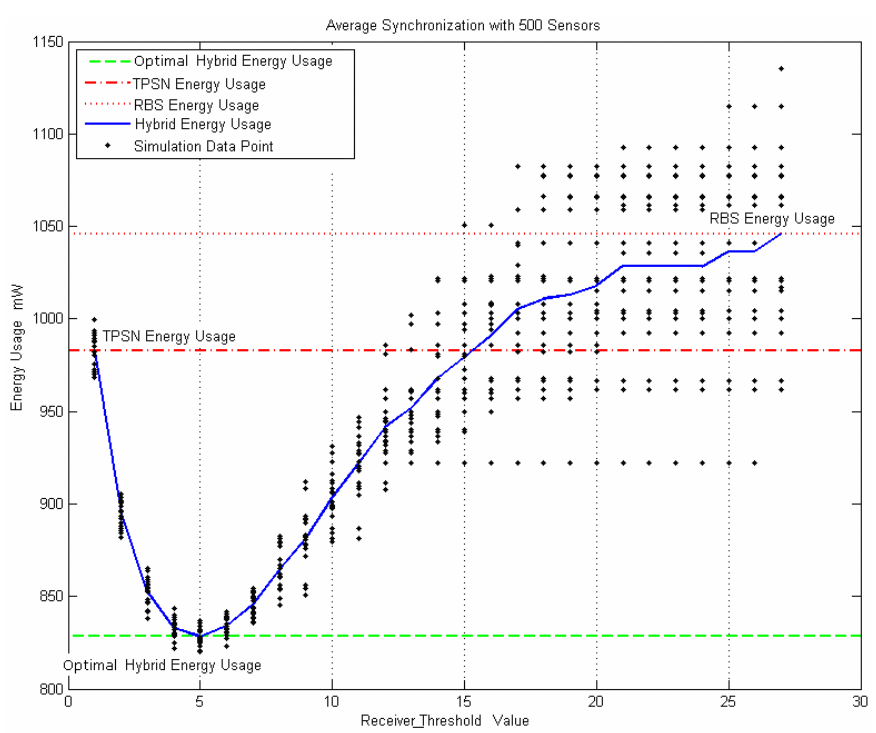

Figure 1: Comparison of the energy usage of RBS, TPSN, and our hybrid algorithm for different values of receiver_threshold values on Mica2DOT platform. The energy usage is in $\mathrm{mW}$.

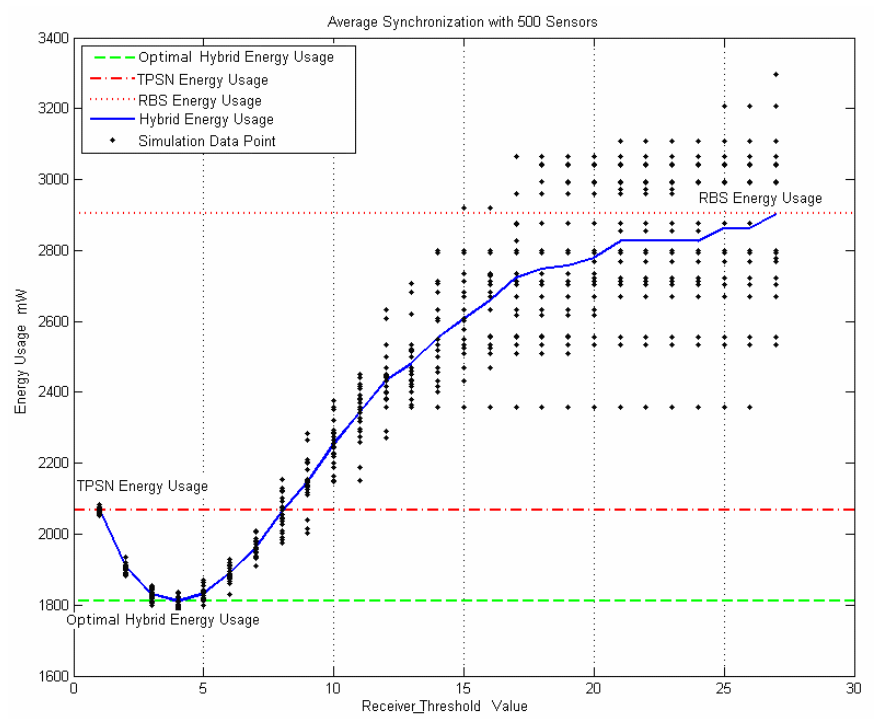

Figure 2: Comparison of the energy usage of RBS, TPSN, and our hybrid algorithm for different values of receiver_threshold values on MicaZ platform. The energy usage is in $\mathrm{mW}$.

The receiver_threshold value is once again changed from 1 to the largest number of receivers connected to a sensor. The hybrid synchronization algorithm is executed for each of these receiver threshold values and the energy consumption 
is stored and compared to the consumption of TPSN, RBS, and the hybrid synchronization algorithm. The results are given in Table I. Table II compares the standard deviations for the energy consumption for each of the algorithms.

Table I: Average energy consumption in $\mathrm{mW}$

\begin{tabular}{|c|c|c|c|c|c|c|}
\hline \# Sensors & $\mathbf{2 5 0}$ & $\mathbf{5 0 0}$ & $\mathbf{7 5 0}$ & $\mathbf{1 0 0 0}$ & $\mathbf{1 2 5 0}$ & $\mathbf{1 5 0 0}$ \\
\hline RBS & 446 & 1046 & 1844 & 2762 & 3756 & 5060 \\
\hline TPSN & 511 & 983 & 1434 & 1885 & 2331 & 2770 \\
\hline Hybrid & 404 & 828 & 1253 & 1672 & 2095 & 2514 \\
\hline $\begin{array}{c}\text { Savings } \\
\text { over RBS }\end{array}$ & $9.29 \%$ & $20.8 \%$ & $32.0 \%$ & $39.4 \%$ & $44.2 \%$ & $50.3 \%$ \\
\hline $\begin{array}{c}\text { Savings } \\
\text { over } \\
\text { TPSN }\end{array}$ & $20.8 \%$ & $15.7 \%$ & $12.7 \%$ & $11.2 \%$ & $10.1 \%$ & $9.2 \%$ \\
\hline
\end{tabular}

Table II: Standard deviation of energy consumption

\begin{tabular}{|c|c|c|c|c|c|c|}
\hline \# Sensors & $\mathbf{2 5 0}$ & $\mathbf{5 0 0}$ & $\mathbf{7 5 0}$ & $\mathbf{1 0 0 0}$ & $\mathbf{1 2 5 0}$ & $\mathbf{1 5 0 0}$ \\
\hline \multirow{2}{*}{ RBS } & 17.3 & 48.0 & 116.9 & 167.8 & 196.6 & 361.4 \\
& $3.90 \%$ & $4.59 \%$ & $6.34 \%$ & $6.07 \%$ & $5.23 \%$ & $7.14 \%$ \\
\hline TPSN & $\begin{array}{c}7.7 \\
1.50 \%\end{array}$ & $\begin{array}{c}8.9 \\
0.90 \%\end{array}$ & $\begin{array}{c}14.3 \\
1.00 \%\end{array}$ & $\begin{array}{c}14.4 \\
0.77 \%\end{array}$ & $\begin{array}{c}18.2 \\
0.78 \%\end{array}$ & $\begin{array}{c}22.1 \\
0.80 \%\end{array}$ \\
\hline Hybrid & $\begin{array}{c}4.0 \\
0.99 \%\end{array}$ & $\begin{array}{c}4.7 \\
0.57 \%\end{array}$ & $\begin{array}{c}5.2 \\
0.42 \%\end{array}$ & $\begin{array}{c}6.9 \\
0.41 \%\end{array}$ & $\begin{array}{c}6.3 \\
0.30 \%\end{array}$ & $\begin{array}{c}6.2 \\
0.27 \%\end{array}$ \\
\hline
\end{tabular}

These results show that RBS's energy consumption is more dependent on the density of sensors in a given area. In contrast, TPSN and the hybrid algorithm are less affected by the size of the network.

When the network size increases from 250 sensors to 500 sensors (for the same area of $1 \mathrm{~km}^{2}$ ), RBS becomes less energy efficient than TPSN. The hybrid algorithm outperforms TPSN by $15.7 \%$, while outperforming RBS by $20.8 \%$. Once the network increases to 750 sensors, RBS clearly becomes less efficient than TPSN. The hybrid algorithm still outperforms TPSN by $12.7 \%$. Since RBS consumes more energy, the hybrid algorithm now outperforms it by $32 \%$.

As more sensors are introduced into the network, RBS becomes dramatically less feasible for a wireless sensor network. As shown in Table I, the hybrid algorithm's energy savings over RBS increases from 39\% with 1000 sensors to over 50\% when the network uses 1500 sensors. In contrast, as the network becomes large, the hybrid algorithm mimics TPSN's behavior, but uses less energy. The energy savings over TPSN are 11\% with 1000 sensors and 9\% with 1500 sensors. For extremely large networks (10,000+ sensors) TPSN has the same efficiency as our proposed algorithm.

\section{CONCLUSIONS}

Wireless sensor networks have tremendous advantages for monitoring object movement and environmental properties but require some degree of synchronization to achieve the best results. The hybrid synchronization algorithm was designed to switch between Timing-sync Protocol for Sensor
Networks (TPSN) and the Reference Broadcast Synchronization algorithm (RBS). These two algorithms allow all the sensors in a network to synchronize themselves within a few microseconds of each other, while at the same time using the least amount of energy possible. The savings in energy varies upon the density of the sensors as well as the reception-to-transmission ratio of energy usage; networks which are saturated with sensors, for example 1500 sensors in a $1 \mathrm{~km}^{2}$ area, will favor TPSN over RBS. TPSN also becomes more favorable as receptions consume more power.

The hybrid algorithm compromises between both of these previous algorithms. The energy savings over RBS can range from $9.3 \%$ in small networks of 250 sensors, to over $50 \%$ for large networks using 1500 sensors. In contrast, the hybrid algorithm's savings over TPSN range from $20.8 \%$ in the same small networks down to $9 \%$ in the large networks. Furthermore, analysis of the standard deviation for each of the algorithms shows RBS's energy consumption can vary dramatically, from nearly $4 \%$ to over $7 \%$, generally increasing for larger networks. In contrast, the standard deviation for TPSN's energy usage decreases from $1.5 \%$ to less than $1 \%$, generally decreasing for larger networks. The hybrid algorithm's deviation is always less than $1 \%$ and continuously decreases down to $0.3 \%$ as more sensors are used.

\section{REFERENCES}

[1] US Coast Guard Navigation Center, http://www.gps.gov/.

[2] NTP: The Network Time Protocol, http://www.ntp.org/.

[3] J. Elson, L. Girod, D. Estrin. "Fine-Grained Network Time Synchronization using Reference Broadcasts," Proceedings of the Fifth Symposium on Operating Systems Design and Implementation (OSDI 2002), December 2002.

[4] S. Ganeriwal, R. Kumar, M. Srivastava. "Timing Sync Protocol for Sensor Networks," ACM SenSys '03, 2003.

[5] S. Ganeriwal, M. Srivastava, "Timing-sync Protocol for Sensor Networks (TPSN) on Berkeley Motes," NESL, 2003.

[6] F. Sivrikaya, B. Yener, "Time synchronization in sensor networks: a survey," IEEE Network, vol. 18, issue 4, July-Aug. 2004 pp.45- 50.

[7] S. Palchaudhuri, A.K. Saha, D.B. Johnsin, "Adaptive clock synchronization in sensor networks," Third International Symposium on Information Processing in Sensor Networks, 2004. IPSN 2004, April 2004, pp. 340-348.

[8] K. Sun, P. Ning, C. Wang, "Secure and resilient clock synchronization in wireless sensor networks," IEEE Journal on Selected Areas in Communications, vol. 24, issue 2, Feb. 2006, pp.395-408.

[9] L. Qun, D. Rus, "Global clock synchronization in sensor networks," IEEE Trans. On Computers, vol. 55, issue 2, Feb. 2006, pp. 214-226.

[10] L. He; G.S. Kuo, "A Novel Time Synchronization Scheme in Wireless Sensor Networks," Vehicular Technology Conference, 2006. VTC 2006-Spring. IEEE 63rd, vol. 2, 2006, pp. 568-572.

[12] H. Lee; W. Yu; Y. Kwon, "Efficient RBS in Sensor Networks," Third International Conference on Information Technology: New Generations, 2006. ITNG 2006, April 2006, pp. 279-284.

[12] Crossbow MICA2Dot Wireless Microsensor Mote, Document Part Number 6020-0043-05 Rev A, http:/www.xbow.com/Products/ Product_pdf_files/Wireless_pdf/MICA2DOT_Datasheet.pdf.

[13] Crossbow MicaZz Wireless Measurement System, Document Part Number 6020-0060-03 Rev A, http://www.xbow.com/Products/ Product_pdf_files/Wireless_pdf/MICAz_Datasheet.pdf 